\title{
Exposed necrotic bone in 183 patients with bisphosphonate-related osteone- crosis of the jaw: Associated clinical characteristics
}

\author{
Leticia Bagan ${ }^{1}$, Yolanda Jiménez ${ }^{2}$, Manuel Leopoldo ${ }^{3}$, Judith Murillo-Cortes ${ }^{4}$, Jose Bagan ${ }^{5}$ \\ ${ }^{1}$ Assistant Professor of Oral Medicine. Universidad Europea de Valencia \\ ${ }^{2}$ Associate Professor of Oral Manifestation of Systemic Diseases. Valencia University \\ ${ }^{3}$ Oral and Maxillofacial Surgeon. Consultant. University General Hospital \\ ${ }^{4}$ University General Hospital, Valencia, Spain \\ ${ }^{5}$ Professor of Oral Medicine, Valencia University. Head Service of Stomatology and Maxillofacial Surgery, University General \\ Hospital. Fundación de Investigación del Hospital General Universitario of Valencia
}

Correspondence:

Service of Stomatology and Maxillofacial Surgery

Avda/ Tres Cruces $s / n$

46014 Valencia, Spain

bagan@uv.es

\begin{abstract}
Bagan L, Jiménez Y, Leopoldo M, Murillo-Cortes J, Bagan J. Exposed necrotic bone in 183 patients with bisphosphonate-related osteonecrosis of the jaw: Associated clinical characteristics. Med Oral Patol Oral Cir Bucal. 2017 Sep 1;22 (5):e582-5.

http://www.medicinaoral.com/medoralfree01/v22i5/medoralv22i5p582.pdf
\end{abstract}

Received: 07/02/2017

Accepted: 07/08/2017

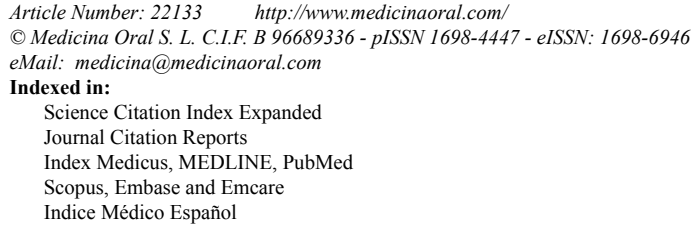

\begin{abstract}
Background: The main objective of our study was to identify oral symptoms and signs most likely to be associated with the exposure of necrotic bone in bisphosphonate-related osteonecrosis of the jaw (BRONJ).

Material and Methods: The study group consisted of 183 patients with BRONJ. We recorded data on the underlying disease, bisphosphonate used, location of osteonecrosis, symptoms, pain, fistula development, suppuration, infection, exposed necrotic bone, and BRONJ stage.

Results: The mean age of the patients was $68.22 \pm 12.19$ years. The sample included $118(64.5 \%)$ women. Breast cancer and multiple myeloma were the most common underlying diseases, and 50 patients received oral bisphosphonates for osteoporosis. Dental extractions (69.4\%) and mandibular location (74.3\%) predominated. The only two variables influencing the possibility of necrotic bone exposure were intravenous bisphosphonate administration and the presence of an intraoral fistula $(p<0.05)$.

Conclusions: Intravenous bisphosphonate use and intraoral fistula presence were associated with a major predisposition to bone exposure in patients with BRONJ.
\end{abstract}

Key words: Bisphosphonate, Osteonecrosis, Jaw.

\section{Introduction}

In 2003, Marx (1) reported a series of 36 cases of osteonecrosis of the jaw following bisphosphonate treatment for cancer. Since then, many other authors have published case series and examined the etiological factors implicated in this severe oral complication, initially known as bisphosphonate-related osteonecrosis of the jaw (BRONJ) (2-8).

The scientific medical literature contains descriptions of risk factors for osteonecrosis $(9,10)$. These risk factors include medication-dependent factors, the most important of which is the timing of bisphosphonate 
treatment. The concomitant use of other drugs, such as corticosteroids and antiangiogenic drugs, increases the risk of osteonecrosis (11). Local factors, such as tooth extraction (12-15), pre-existing periodontal problems, and implant or dental prosthesis use, can also predispose patients to BRONJ (9).

Clinically, exposed areas of necrotic bone may be observed; in other cases, only fistulas to the bone are detected. In stage 0 BRONJ, communication between the oral mucosa and the necrotic bone is absent $(11,16-18)$. Information on factors determining the presence of bone exposure is scarce. The main objective of our study was to identify the local oral symptoms and signs associated most frequently with the exposure of bone in BRONJ.

\section{Material and Methods}

This retrospective study was performed at the Department of Stomatology and Maxillofacial Surgery, University General Hospital, Valencia, Spain, between September 2005 and December 2015. The study group consisted of 183 patients with BRONJ. The inclusion criteria were: (1) presence of at least one area of osteonecrosis on the mandible or maxilla and (2) diagnosis of BRONJ according to the criteria established by Ruggiero et al. (11).

We recorded data on the underlying disease, medications used, mode of bisphosphonate administration (oral or intravenous), and timing of medication use. We also collected data on patients' symptoms, pain, presence of fistula, suppuration, infection, presence of exposed necrotic bone, and location of osteonecrosis on the mandible or maxilla. We determined the BRONJ stage in each case according to the criteria of Ruggiero et al. (11).

For the statistical analysis, we calculated means and percentages for the study variables. We performed a logistic regression analysis to identify variables influencing the dependent variable, which was the presence or absence of bone exposure. We considered $p<0.05$ to indicate statistical significance.

\section{Results}

Table 1 shows the clinical findings for the 183 patients. The sample comprised 118 (64.5\%) women and 65 $(35.5 \%)$ men with a mean age of $68.22 \pm 12.19$ years.

The most frequent underlying diseases were breast cancer and multiple myeloma. Forty-seven patients were treated with oral bisphosphonates due to osteoporosis. Zoledronic acid was the most common treatment (72.1\%). The mean duration of intravenous bisphosphonate therapy was $24.42 \pm 19.27$ months, and that of oral bisphosphonate therapy was $85.36 \pm 54.97$ months.

The most prevalent local predisposing factor for osteonecrosis development was dental extraction (69.4\%), and mandibular location predominated ( $74.3 \%$ of sites). Pain was the most frequently documented symptom
(64.5\%), followed by suppuration (48.6\%), intraoral fistula (35.5\%), inflammation (33.9\%), and extraoral fistula $(7.7 \%)$. Bone exposure was present in $78.1 \%$ of cases. Stage 2 was the most common stage of osteonecrosis $(46.4 \%)$.

In the logistic regression analysis, necrotic bone exposure served as the dependent variable and age, sex, local factors, osteonecrosis location, underlying disease, mode of bisphosphonate administration (intravenous or oral), and the symptoms and signs described above (pain, intraoral fistula, extraoral fistula) served as independent variables. The only two variables that influenced the possibility of necrotic bone exposure were the mode of bisphosphonate administration (intravenous or oral) and the presence of intraoral fistula $(p<0.05)$.

\section{Discussion}

BRONJ is more common in women, due mainly to the large number of cases associated with treatment of breast cancer and osteoporosis (19). In our sample, 118 of $183(64.5 \%)$ patients were female, and $55.3 \%$ of the sample had breast cancer or osteoporosis.

The mean age of patients with osteonecrosis is $>65$ years $(20,21)$. In agreement with this finding, the mean age of our patients was $68.22 \pm 12.19$ years (range: 32 89 years).

The risk of osteonecrosis is greater in oncologic patients receiving intravenous treatment than in those receiving oral bisphosphonates. Furthermore, the risk of osteonecrosis in patients with cancer treated with zoledronic acid is 50 to 100 times greater than that associated with placebo. The most common malignant diseases for which bisphosphonates are typically used are breast cancer, lung cancer, prostate cancer, and multiple myeloma (11). The most frequent disease in our series was breast cancer $(30.1 \%)$, followed by multiple myeloma (26.20\%). The percentages for other diseases, such as prostate cancer $(12 \%)$, lung cancer $(2.2 \%)$, and kidney cancer $(2.2 \%)$, were much lower. Patients with osteoporosis comprised $25.7 \%$ of the sample, and all of the patients were women.

Most (72.1\%) patients in our sample had been treated with zoledronic acid; $27.3 \%$ of patients had received oral bisphosphonates, such as alendronate (17,5\%), ibandronate (6.6\%), and risedronate (3.3\%).

In our series, the duration of intravenous and oral bisphosphonate treatment was $25.42 \pm 19.27$ and $85.36 \pm$ 54.97 months, respectively. The duration of oral bisphosphonate treatment greatly exceeded that recommended by experts.

The most common local predisposing factor for osteonecrosis is dental extraction, with $52-61 \%$ of patients reporting previous extraction due to periodontal or periapical infection $(22,23)$. Our findings are in agreement with the majority of the published data; we found that 
Table 1: Clinical data for 183 cases of bisphosphonate-related osteonecrosis of the jaw.

\begin{tabular}{|c|c|c|c|}
\hline & Type & Frecuency & Porcentage \\
\hline \multirow[t]{9}{*}{ Type of disease } & Breast cancer & 55 & $30,1 \%$ \\
\hline & Prostate cancer & 22 & $12,0 \%$ \\
\hline & Lung cancer & 4 & $2,2 \%$ \\
\hline & Renal cancer & 4 & $2,2 \%$ \\
\hline & Colon cancer & 1 & $0,5 \%$ \\
\hline & Breast cancer and Multiple myeloma & 1 & $0,5 \%$ \\
\hline & Multiple myeloma & 48 & $26,2 \%$ \\
\hline & Follicular lymphoma & 1 & $0,5 \%$ \\
\hline & Osteoporosis & 47 & $25,7 \%$ \\
\hline \multirow[t]{4}{*}{ Type of drug } & Zoledronic acid & 132 & $72,1 \%$ \\
\hline & Alendronate & 32 & $18 \%$ \\
\hline & Ibandronate & 12 & $6,6 \%$ \\
\hline & Risedronate & 6 & $3,3 \%$ \\
\hline \multirow[t]{2}{*}{ Bisphosphonate } & Intravenous & 133 & $72,7 \%$ \\
\hline & Oral & 50 & $27,3 \%$ \\
\hline \multirow[t]{6}{*}{ Local factors } & Dental extraction & 127 & $69,4 \%$ \\
\hline & Prosthesis & 17 & $9,3 \%$ \\
\hline & Implant & 9 & $4,9 \%$ \\
\hline & Spontaneous & 7 & $3,8 \%$ \\
\hline & Extraction and prosthesis & 1 & $0,6 \%$ \\
\hline & Unknown & 22 & $12 \%$ \\
\hline \multirow[t]{3}{*}{ Location } & Mandible & 136 & $74,3 \%$ \\
\hline & Mandible and maxilla & 17 & $9,3 \%$ \\
\hline & Maxilla & 30 & $16,4 \%$ \\
\hline Pain & Yes & 118 & $64,5 \%$ \\
\hline Inflammation & Yes & 62 & $33,9 \%$ \\
\hline Suppuration & Yes & 89 & $48,6 \%$ \\
\hline Intraoral fistula & Yes & 65 & $35,5 \%$ \\
\hline Extraoral fistula & Yes & 14 & $7,7 \%$ \\
\hline Bone exposure & Yes & 143 & $78,1 \%$ \\
\hline \multirow{4}{*}{$\begin{array}{c}\text { Stanging } \\
\text { classification }\end{array}$} & 0 & 8 & $4,4 \%$ \\
\hline & 1 & 49 & $26,8 \%$ \\
\hline & 2 & 85 & $46,4 \%$ \\
\hline & 3 & 41 & $22,4 \%$ \\
\hline
\end{tabular}

dental extraction was a predisposing local factor in $69.4 \%$ of cases, followed by prosthesis use $(9.3 \%)$, implant placement (4.9\%), and spontaneous development or unknown cause $(3.8 \%)$.

Osteonecrosis is known to occur more frequently in the mandible $(74.3 \%)$ than in the maxilla (16.4\%). However, both locations have been affected in a few reported cases (24). Most (74.3\%) of our cases were mandibular, while $16.4 \%$ of patients presented with maxillary le- sions, and 9.3\% showed osteonecrosis in both locations. These percentages are similar to those reported by Kos et al. (24).

In 2007, Marx (25) reported a direct correlation between reported pain and clinical evidence of infection. In our series, $35.5 \%$ of patients reported no pain; these cases were classified as stage 1 . The remaining $64.5 \%$ of patients reported pain, which was usually caused by associated infection (25). 
In recent years, several modifications have been made to the osteonecrosis staging system. Ruggiero et al. (3) proposed one of the first classifications in 2006. In 2009, stage 0 was added to the classification, with the aim of including patients with nonspecific symptoms, such as pain, and those with other poorly defined clinical and radiographic alterations (26). In 2014, the classification was updated, with the addition of clinical features such as the presence of fistula to the bone in stages 1-3 (11). The presence of necrotic bone exposure is the main clinical feature in the most recent classification. We found this feature in $78.1 \%$ of our patients. In our series, $4.4 \%$ of patients had stage $0,26.8 \%$ had stage $1,46.4 \%$ had stage 2 , and $22.4 \%$ had stage 3 disease.

The main objective of this study was to identify other clinical symptoms and signs influencing the presence of bone exposure in BRONJ using logistic regression. We found that the use of intravenous bisphosphonates and the presence of intraoral fistula were associated with a major predisposition to the development of bone exposure. From a clinical perspective, these features are the most significant of those examined in this study.

\section{References}

1. Marx RE. Pamidronate (Aredia) and zoledronate (Zometa) induced avascular necrosis of the jaws: a growing epidemic J Oral Maxillofac Surg. 2003;61:1115-7.

2. Bagan JV, Murillo J, Jimenez Y, Poveda R, Milian MA, Sanchis $\mathrm{JM}$, et al. Avascular jaw osteonecrosis in association with cancer chemotherapy: series of 10 cases J Oral Pathol Med. 2005;34:120-3. 3. Ruggiero SL, Fantasia J, Carlson E. Bisphosphonate-related osteonecrosis of the jaw: background and guidelines for diagnosis, staging and management. Oral Surg Oral Med Oral Pathol Oral Radiol Endod. 2006;102:433-41.

4. Boonyapakorn T, Schirmer I, Reichart PA, Sturm I, Massenkeil G. Bisphosphonate-induced osteonecrosis of the jaws: prospective study of 80 patients with multiple myeloma and other malignancies. Oral Oncol. 2008;44:857-69.

5. Lazarovici TS, Yahalom R, Taicher S, Elad S, Hardan I, Yarom N.Bisphosphonate-related osteonecrosis of the jaws: a single-center study of 101 patients. J Oral Maxillofac Surg. 2009;67:850-5.

6. Bagan J, Sheth CC, Soria JM, Margaix M, Bagan L. Bisphosphonates-related osteonecrosis of the jaws: a preliminary study of salivary interleukins. J Oral Pathol Med. 2013;42:405-8.

7. Vescovi P, Merigo E, Meleti M, Manfredi M, Guidotti R, Nammour S. Bisphosphonates-related osteonecrosis of the jaws: a concise review of the literature and a report of a single-centre experience with 151 patients. J Oral Pathol Med. 2012;41:214-21.

8. Vescovi P, Meleti M, Merigo E, Manfredi M, Fornaini C, Guidotti $\mathrm{R}$, et al. Case series of 589 tooth extractions in patients under bisphosphonates therapy. Proposal of a clinical protocol supported by Nd:YAG low-level laser therapy. Med Oral Patol Oral Cir Bucal. 2013;18:e680-5.

9. Japanese Allied Committee on Osteonecrosis of the Jaw, Yoneda T, Hagino H, Sugimoto T, Ohta H, Takahashi S, et al. Antiresorptive agent-related osteonecrosis of the jaw: Position Paper 2017 of the Japanese Allied Committee on Osteonecrosis of the Jaw. J Bone Miner Metab. 2017;35:6-19.

10. Fung P, Bedogni G, Bedogni A, Petrie A, Porter S, Campisi G, et al. Time to onset of bisphosphonate-related osteonecrosis of the jaws: a multicentre retrospective cohort study. Oral Dis. 2017;23:477-483. 11. Ruggiero SL, Dodson TB, Fantasia J, Goodday R, Aghaloo T, Mehrotra B, et al. American Association of Oral and Maxillofacial
Surgeons position paper onmedication-related osteonecrosis of the jaw--2014 update. J Oral Maxillofac Surg. 2014;72:1938-56.

12. Bagan JV, Jimenez Y, Murillo J, Hernandez S, Poveda R, Sanchis $\mathrm{JM}$, et al. Jaw osteonecrosis associated with bisphosphonates: multiple exposed areas and its relationship to teeth extractions. Study of 20 cases. Oral Oncol. 2006;42:327-9.

13. Lodi G, Sardella A, Salis A, Demarosi F, Tarozzi M, Carrassi A. Tooth extraction in patients taking intravenous bisphosphonates: a preventive protocol and case series. J Oral Maxillofac Surg. 2010;68:107-10.

14. Malden N, Beltes C, Lopes V. Dental extractions and bisphosphonates: the assessment, consent and management, a proposed algorithm. Br Dent J. 2009;206:93-8.

15. Yamazaki T, Yamori M, Ishizaki T, Asai K, Goto K, Takahashi $\mathrm{K}$, et al. Increased incidence of osteonecrosis of the jaw after tooth extraction in patients treated with bisphosphonates: a cohort study. Int J Oral Maxillofac Surg. 2012;41:1397-403.

16. Nicolatou-Galitis O, Papadopoulou E, Sarri T, Boziari P, Karayianni A, Kyrtsonis MC, et al. Osteonecrosis of the jaw in oncology patients treated with bisphosphonates: prospective experience of a dental oncology referral center. Oral Surg Oral Med Oral Pathol Oral Radiol Endod. 2011;112:195-202.

17. Fedele S, Porter SR, D’Aiuto F, Aljohani S, Vescovi P, Manfredi $\mathrm{M}$, et al. Nonexposed variant of bisphosphonate-associated osteonecrosis of the jaw: a case series. Am J Med. 2010;123:1060-4.

18. Bedogni A, Fedele S, Bedogni G, Scoletta M, Favia G, Colella G, et al. Staging of osteonecrosis of the jaw requires computed tomography for accurate definition of the extent of bony disease. Br J Oral Maxillofac Surg. 2014;52:603-8.

19. Black DM, Reid IR, Boonen S. The effect of 3 versus 6 years of zoledronic acid treatment of osteoporosis: A randomized extension to the HORIZON-Pivotal Fracture Trial (PFT). J Bone Miner Res. 2012;27:243-54.

20. Stumpe MR, Chandra RK, Yunus F. Incidence and risk factors of bisphosphonate associated osteonecrosis of the jaws. Head Neck. 2009;31:202-6.

21. Saad F, Brown JE, Van Poznak C. Incidence, risk factors, and outcomes of osteonecrosis of the jaw: Integrated analysis from three blinded active-controlled phase III trials in cancer patients with bone metastases. Ann Oncol. 2012;23:1341-7.

22. Khan AA, Morrison A, Hanley DA, Felsenberg D, McCauley LK, O'Ryan F, et al .Diagnosis and management of osteonecrosis of the jaw: a systematic review and international consensus. J Bone Miner Res. 2015;30:3-23.

23. Kos M. Association of dental and periodontal status with bisphosphonate-related osteonecrosis of the jaws. A retrospective case controlled study. Arch Med Sci. 2014;10:117-23.

24. Kos M. Incidence and risk predictors for osteonecrosis of the jaw in cancer patients treated with intravenous bisphosphonates. Arch Med Sci. 2015;11:319-24

25. Marx RE, Cillo JE Jr, Ulloa JJ. Oral bisphosphonate-induced osteonecrosis: risk factors, prediction of risk using serum CTX testing, prevention, and treatment. J Oral Maxillofac Surg. 2007;65:2397410 .

26. Ruggiero SL, Dodson TB, Assael LA, Landesberg R, Marx RE, Mehrotra B, et al. American Association of Oral and Maxillofacial Surgeons position paper on bisphosphonate-related osteonecrosis of the jaw - 2009 update. Aust Endod J. 2009;35:119-30.

\section{Conflict of Interest}

None to declare. 